ARCHIVO ESPAÑOL DE ARTE, LXXXVII, 346

ABRIL-JUNIO 2014, pp. 171-178

ISSN: 0004-0428, eISSN: 1988-8511

doi: $10.3989 /$ aearte.2014.13

\title{
LOS PEROLLI EN LA MANCHA: NUEVAS APORTACIONES
}

\author{
JOSÉ JAVIER BARRANQUERO CONTENTO
}

\begin{abstract}
Este trabajo aporta nuevos datos sobre la labor que desarrollaron dos miembros de la familia Perolli, Esteban y Juan Esteban, bajo el patrocinio del II Marqués de Santa Cruz, don Álvaro de Bazán y Benavides.

Palabras clave: Esteban Perolli; Juan Esteban Perolli; Álvaro de Bazán y Benavides; Palacio; Viso del Marqués; Nuestra Señora de las Nieves; Almagro.
\end{abstract}

\section{THE PEROLLI IN LA MANCHA: NEW CONTRIBUTIONS}

This study offers new data on the work carried out by two members of the Perolli family, Esteban and Juan Esteban, under the patronage of the second marquis of Santa Cruz, Don Álvaro de Bazán y Benavides.

Key words: Esteban Perolli; Juan Esteban Perolli; Álvaro de Bazán y Benavides; Palace; Viso del Marqués; Nuestra Señora de las Nieves; Almagro.

Los Perolli desempeñaron un papel muy importante en la configuración del panorama artístico de La Mancha durante el último tercio del siglo XVI y principios del XVII. Rosa López Torrijos dedicó dos exhaustivos estudios a la etapa italiana de Juan Bautista Perolli, recogiendo en el primero de ellos todas las noticias que se habían publicado hasta el momento sobre los miembros de esta familia ${ }^{1}$. Entre los trabajos que se han realizado analizando la obra de los Perolli en La Mancha tendríamos que mencionar el clásico de Agustín Bustamante y Fernando Marías $^{2}$, y otros más recientes como los de Eduardo Blázquez Mateos ${ }^{3}$, Enrique Herrera Maldonado $^{4}$ o la propia Rosa López Torrijos 5 .

A la vista de este amplio panorama historiográfico, no cabe duda de que se ha avanzado mucho en el conocimiento de esta familia, pero lo cierto es que todavía queda bastante por decir sobre ella y, precisamente, dentro de este contexto, nuestro modesto trabajo aporta varios datos inéditos que ayudan a precisar su actividad dentro del complejo panorama artístico de la época.

Las dos primeras referencias que podemos aportar tienen que ver con Juan Esteban Perolli 6 y la más antigua de ellas aparece en la carta de poder que otorgaron Juan Bautista Perolli, hijo,

1 López ToRriJos, 2000: 1-22; 2002: 145-165.

2 Bustamante y Marías, 1982: 173-185.

3 Blázquez Mateos, 1999.

4 Herrera Maldonado, 2003: 259-271.

5 LÓPEZ TORRIJOS, 2004: 129-138; 2005: 51-59; 2007: 198-202; 2009.

6 Juan del Campo Muñoz afirmó en su momento que Juan Esteban era hijo de Juan Bautista Perolli, pero nosotros pensamos que esta conclusión no es del todo acertada porque, si repasamos los datos aportados en su trabajo, nos damos 
y Lucrecia Perolli el 7 de abril de 1600. En este documento, por el que los dos hijos de Juan Bautista cedían a Juan Ruiz de Elvira el Mozo el derecho a cobrar la cantidad que la iglesia y el concejo de Manzanares adeudaban a su difunto padre por la construcción de la sacristía, aparecen como testigos tres personajes que tenían algo en común, su condición de artistas. Se trata de Juan Bautista el Viejo, albañil; maese Francisco, cantero, y Juan Esteban de Perolis ${ }^{7}$. Por desgracia el documento no nos ofrece ningún dato más sobre estos tres maestros, pero es obvio que este Juan Esteban no era el hijo de Juan Bautista porque, si lo hubiera sido, habría comparecido como otorgante y no como testigo.

El segundo testimonio que hemos localizado sobre Juan Esteban tiene una enorme importancia desde el punto de vista artístico y, como no podía ser de otra forma, estaría relacionado con el Palacio de don Álvaro de Bazán en El Viso del Marqués, edificio que marcó el inicio de la actividad de esta familia en España. Se trata de una anotación que aparece en las cuentas que otorgaron Gaspar de Garnica, Sebastián Ruiz de Ocaña y Juan Pérez, como administradores de los dominios del Marqués, ante Juan Sáenz de Oyanguren, contador de don Álvaro, y Baltasar Calderón, escribano público de Almagro, el 22 de mayo de 1604. En el apartado dedicado al pago de salarios y raciones que se dan a gobernadores y criados viejos de su exelencia y otras personas en el dicho Estado el año de 1603, y bajo el epígrafe de Salario al pintor nos encontramos con una anotación en la que se detallan los pagos que se realizaron a Juan Esteban Perolli. En concreto, el documento afirma lo siguiente:

Mas se le reciven en quenta veynte y siete doçientos (sic.) y sesenta y ocho maravedis que pago a Joan Estevan de Perola pintor en esta manera los quinientos y cinquenta reales en dinero y los 8.568 maravedis por diez y ocho fanegas de trigo en grano que le dieron a catorce reales como a valido el de el dicho año de 603 y con los dichos 550 reales fue pagado del salario de un

\footnotetext{
cuenta de que confunde o, mejor dicho, adjudica a una misma persona datos que en realidad se corresponderían con dos hombres que llevaban el mismo nombre. En efecto, el autor recoge la existencia de dos hijos del maestro que se llamaban Juan, bautizados respectivamente en 1581 y 1584, y también menciona un bautizo, celebrado el 16 de diciembre de 1593, en el que actuaron como compadres Juan Esteban de Perolli y Jerónima Pasano, su madre. Sin embargo, unas líneas después, el autor afirma que las primeras noticias que consiguió recoger sobre Juan Esteban datan del 15 de octubre de 1584, fecha en la "que consta como padrino con Jerónima de Perola, mujer de Juan Bautista Perola, de una niña -Catalina- cuyos padres son un Marco Antonio, italiano, e Isabel Hernández". Resulta cuando menos chocante que un niño que como mucho podría tener tres años actuase como padrino de otra niña, pero los datos contradictorios no terminan aquí. Posteriormente, Juan del Campo recoge también la fecha de bautizo de los hijos que tuvo Juan Esteban con Juana de Ribadeneyra, su mujer, y curiosamente, el primero, un niño llamado también Juan, recibió las aguas el 26 de agosto de 1594 (Del Campo Muñoz, 1998: 57-58). Es obvio que un hombre nacido en 1581 o 1584 no podría tener un hijo diez años después. Estamos, por tanto, ante dos personas que se llamaban Juan Esteban y que pertenecían a dos generaciones distintas de la misma familia. El más joven sí sería hijo de Juan Bautista, pero el de más edad mantendría con éste un parentesco que todavía está por determinar, aunque bien pudiera ser su hermano. Lógicamente, el Juan Esteban al que nos vamos a referir en este artículo sería el más viejo y formaría parte de esa tercera maestranza que llegó al Viso de la mano de Juan Bautista. Las dificultades a la hora de determinar la verdadera identidad de cada uno de los miembros de esta familia estaría relacionada con la costumbre de poner a los hijos el nombre de los padres o los tíos. Un buen ejemplo de esto que acabamos de decir sería el nombre completo de esos dos hijos de Juan Bautista que fueron bautizados como Juan. En realidad, uno de ellos se llamaba Juan Esteban, como hemos tenido ocasión de analizar, y el otro Juan Bautista, como su padre, tal y como se desprende de la carta de poder que otorgó este personaje, junto a su hermana Lucrecia, el 7 de abril de 1600. Traslado de la carta de poder otorgada por Juan Bautista Perolis y Lucrecia de Perolis, su hermana, el 7 de abril de 1600, ante Silvestre de Moya, escribano público de El Viso del Marqués. Archivo Histórico Nacional (AHN), Órdenes Militares (OOMM), Archivo Histórico de Toledo, legajo 39137, sin foliar (sf).

7 Traslado de la carta de poder otorgada por Juan Bautista Perolis y Lucrecia de Perolis, su hermana, el 7 de abril de 1600, ante Silvestre de Moya, escribano público de El Viso del Marqués. AHN, OOMM, Archivo Histórico de Toledo, legajo 39137, sf. El Juan Bautista el Viejo que aparece en este documento sería Juan Bautista Casella.
}

Arch. esp. arte, LXXXVII, 346, ABRIL-JUNIO 2014, 171-178 ISSN: 0004-0428, eISSN: 1988-8511, doi: 10.3989/aearte.2014.13 
año que a de haver en dinero y con las diez y ocho fanegas de trigo de un año del que se le manda dar en cada uno como parece de tres cartas de pago del susodicho de 18 de otubre del dicho año de 603 y de 21 de diziembre del y otra de 9 de abril de $604^{8}$.

A nuestro juicio, el testimonio que acabamos de aportar estaría directamente relacionado con la ejecución de las pinturas del Palacio. El hecho de tener contratado a un pintor, al que se le pagaba un salario de forma regular, es un indicio lo suficientemente relevante como para poder afirmar que todavía estaban realizándose los frescos, aunque en el asiento no se haga referencia a la tarea que estaba haciendo Juan Esteban. El documento, por tanto, nos coloca ante dos cuestiones fundamentales, como son la autoría de las pinturas y la datación de las mismas. Tal como expone Rosa López Torrijos, Juan Bautista Perolli aparece en las cuentas del Palacio cobrando por su labor como pintor los años 1579 y 1580 , año éste último en el que pudo hacerse cargo de la dirección de las obras tras la muerte del maestro Domingo Casella9. Juan Bautista, por su parte, falleció en 1586; de ahí que la referencia que aportamos más arriba nos permite certificar dos cosas en relación con la decoración del Palacio, que Juan Esteban se había hecho cargo de la ornamentación del edificio y que la tarea de pintarlo se extendió al menos hasta 1604, aunque es bastante probable que las labores continuasen después de esta fecha porque en las cuentas no se hace referencia a que, con ese pago, terminase la relación contractual entre el artista y el Marqués ${ }^{10}$. Determinar el momento en el que Juan Esteban comenzó a ser el pintor del Palacio, entendiendo por este término el de maestro que estaba al frente de la realización de los frescos, es imposible con los datos de que disponemos, pero lo más lógico es pensar que pasara a desempeñar esta función tras la muerte de Juan Bautista.

Por otra parte, la posibilidad de que todavía se estuvieran realizando intervenciones de cierta envergadura en el Palacio quedaría corroborada por otros dos asientos de las cuentas en los que se recoge el pago de 204.000 maravedís para gastos y reparos del mismo ${ }^{11}$. Las cantidades invertidas en este apartado debían ser tan importantes que los administradores presentaron una alegación a las cuentas en la que se quejaban de esta situación y dejaban claro que hacer frente a este tipo de gastos no era tarea suya ${ }^{12}$.

${ }^{8}$ Cuentas otorgadas por Gaspar de Garnica y consortes como administradores del Estado, rentas y encomienda del Marqués de Santa Cruz ante Baltasar Calderón, el 22 de mayo de 1604. Archivo Histórico Provincial de Ciudad Real (AHPCR), Protocolos, legajo 1613, sf.

9 La autora se hace eco de las referencias de Ponz, Ceán y Alcalá Galiano en las que se afirma que Juan Bautista Prioli era el Maestro Mayor de las obras en 1585. Sin embargo, considera que debió desempeñar esta función desde la muerte de Domingo. LÓPEZ TORRIJOS, 2009: 208.

${ }^{10}$ No compartimos, por tanto, las opiniones de Enrique Herrera sobre la construcción y decoración del Palacio. Este autor precisa que los frescos se terminaron en 1586, coincidiendo con la muerte de Juan Bautista. Para afirmar esto se apoya en una inscripción que aparece "en una cartela pintada en la bóveda del segundo piso de la panda oeste del patio" en la que puede leerse " $A C A B O S E E L A N T O M C D X X X V T$ ". Dejando a un lado el hecho de que la inscripción no indica el año mencionado por este investigador, lo que sin duda se debe a una pequeña confusión, Enrique Herrera también sostiene que, tras el fallecimiento de Juan Bautista, Esteban se hizo cargo de la maestría del Palacio. HerRERA MALDONADO, 2003: 265.

${ }^{11}$ En un primer asiento se recogen ciento y treynta y seis mil maravedis que en dos veces a dos mill reales cada una an pagado a Anto (sic.) Franco mayordomo de su exelencia para gastos de las casas de Palacio del Viso como parece de su carta de pago una de ocho de hebrero y otra de nueve de abrill de 604. Poco después se menciona una nueva anotación en la que se dejaba constancia del pago de otros dos mill reales que se dieron al dicho Anton Franco para los reparos de la dicha casa de Palacio del Viso. Cuentas otorgadas por Gaspar de Garnica y consortes como administradores del Estado, rentas y encomienda del Marqués de Santa Cruz ante Baltasar Calderón, el 22 de mayo de 1604. AHPCR, Protocolos, legajo 1613, sf.

12 Los administradores precisaron que no enbargante que para el rreparo de las cassas del Visso que su exelencia tiene prinzipales en ella an dado los maravedis que an sido nescesarios para el dicho rreparo segun los a pedido Anton

Arch. esp. arte, LXXXVII, 346, ABRIL-JUNIO 2014, 171-178

ISSN: 0004-0428, eISSN: 1988-8511, doi: 10.3989/aearte.2014.13 
Las cuentas, además, nos permiten valorar la importancia que se concedía a la tarea desarrollada por un artista de la reputación de Juan Esteban Perolli, al poder comparar su salario con el que recibían otras personas vinculadas al Marqués de Santa Cruz que desempeñaban cargos institucionales. Este es el caso de Antón Franco, mayordomo de rentas del Marqués, que cobraba 40.000 maravedís; del capitán Francisco de las Cabezas, gobernador de las villas de Santa Cruz de Mudela y de El Viso del Marqués, que recibía 35.000 maravedís; o del doctor Gregorio López, gobernador de Valdepeñas, que percibía 50.000. El párroco de esta misma localidad, por su parte, recibía 10.800 maravedís, cantidad a la que habría que añadir el valor del beneficio curado, y el de Santa Cruz de Mudela, 12.000. A la vista de estos datos, podemos decir que el salario de Juan Esteban Perolli (salario que ascendía a 27.268 maravedís) era relativamente importante. Antón Franco cobraba mucho más, pero en esos momentos también ejercía como contador debido a la ausencia de Juan Sáenz de Oyanguren, lo que posiblemente conllevase un incremento del sueldo. No obstante, la comparación que puede resultar más ilustrativa es la que podemos realizar con el salario del doctor Gregorio López, ya que alguien que desempeñaba el cargo de gobernador de una localidad tan relevante como la de Valdepeñas, y que por tanto ostentaba la representación del Marqués en ella, percibía algo menos del doble que el pintor.

Las demás referencias que hemos encontrado sobre esta familia de artistas están relacionadas con Esteban Perolli ${ }^{13}$ y con la construcción (o, mejor dicho, reedificación) de la ermita de Nuestra Señora de las Nieves, patrona de Almagro. La reforma fue costeada por don Álvaro de Bazán y Benavides (II Marqués de Santa Cruz) y, como era de esperar, en la génesis de este proyecto

Franco mayordomo de su exelencia y que daran lo que fuere su voluntad adelante para lo susodicho; que no por esto sea visto ser obligados los susodichos a rrepar (sic.) las dichas cassas ni parte dellas ni dar cossa para ello porque conforme a su asiento no lo estan obligados ni en el se les encarga ni se hace mincion de las dichas casas porque para las rreparar por ser muy grandes y de muncha costa conforme ellas lo avrian menester era nescesario muncha suma de maravedis para lo susodicho y si esto se les pidiera quando se hizo el dicho asiento o lo pusieran en el no lo consintieran como aora no lo consienten sino que sin perjuicio de su derecho yran dando para el dicho rreparo dellas lo que fuere su voluntad sin quedar como no quieren quedar ni quedan obligados a ello por obligazion de fuerça y asi lo protestan. Cuentas otorgadas por Gaspar de Garnica y consortes como administradores del Estado, rentas y encomienda del Marqués de Santa Cruz ante Baltasar Calderón, el 22 de mayo de 1604. AHPCR, Protocolos, legajo 1613, sf.

${ }^{13}$ La figura de Esteban ha planteado no pocos problemas a la historiografía del arte. Del Campo Muñoz afirma que podría tratarse de un hermano o un sobrino de Juan Bautista Perolli, inclinándose más bien por esta segunda posibilidad. El autor recoge una mención, la primera que aporta en torno a este personaje, en la que se afirma que Esteban ejerce como padrino el 17 de septiembre de 1580 en el bautizo de una niña junto a su cuñada, Jerónima Pasano. Posteriormente, en 1588, certifica su matrimonio con Hipólita Pasano, hermana de Jerónima. Hasta aquí ningún problema, porque tendríamos a dos hermanos (Juan Bautista y Esteban) casados con dos hermanas (Jerónima e Hipólita), pero las dificultades surgen el 18 de mayo de 1589, cuando recoge otra referencia en la que se hace mención a Esteban de Peroli como padrino de la hija de un cantero, responsabilidad que compartió con su tía, Jerónima Pasana (DEL CAMPO MUÑOZ, 1998: 56-57). Esta mención entra en clara contradicción con la anterior y el autor pretende resolverla haciendo a Esteban sobrino de Juan Bautista y casándolo con la hermana de Jerónima, matrimonio que a todas luces no nos convence, y no porque no se produjera una boda entre dos personas llamadas Esteban Perolli e Hipólita Pasano, que sí tuvo lugar, sino porque es imposible que Esteban fuera cuñado de Jerónima en 1580, y por tanto hermano de Juan Bautista, y nueve años después pasara a ser sobrino de ésta. Rosa López Torrijos se inclina por pensar que Juan Bautista y Esteban eran hermanos (LÓPEZ TORRIJOS, 2005: 56 y 58; 2009: 196), mientras que Enrique Herrera se inclina por hacerlos tío y sobrino (Herrera Maldonado, 2003: 265-266; 2005: 392). No obstante, y con independencia de su parentesco con Juan Bautista, lo cierto es que tanto Rosa López Torrijos como Enrique Herrera coinciden en afirmar que este personaje se trasladó hasta Almagro donde dio origen a la saga de los Perolas de esta localidad. Nosotros pensamos que, si las transcripciones realizadas por Juan del Campo son correctas, y no dudamos de ello, lo lógico sería pensar que estamos de nuevo ante dos personas distintas que compartían el mismo nombre: uno que era hermano de Juan Bautista y otro que sería sobrino de éste. Los datos que vamos a aportar en este trabajo, por el contexto cronológico al que pertenecen, demasiado tardío, estarían relacionados con la figura del sobrino y no con el hermano.

Arch. esp. arte, LXXXVII, 346, ABRIL-JUNIO 2014, 171-178 ISSN: 0004-0428, eISSN: 1988-8511, doi: 10.3989/aearte.2014.13 
nos volvemos a encontrar con otro miembro de esta saga de artistas que estuvo tan estrechamente vinculada a la casa de Santa Cruz.

La ermita (fig. 1) fue estudiada ya por Clementina Díez de Baldeón que trató de precisar el momento en el que se levantó su fábrica y, al mismo tiempo, determinar la autoría de su diseño, vinculándolo por razones estilísticas con la figura de Nicolás de Vergara el $\mathrm{Mozo}^{14}$. Eduardo Blázquez Mateos también dedicó unas líneas a este edificio y, aunque volvió a repetir las fechas que manejó Diez de Baldeón, aportó una interesante referencia en torno a los maestros que realizaron la obra. El autor menciona una "escritura de la fábrica del edificio", fechada el 13 de febrero de 1629, que aparece en uno de los legajos conservados en el Archivo de Santa Cruz. Este documento le sirvió para determinar que "las obras fueron firmadas y selladas (sic.) por los maestros Juan Martín Chillero, Juan Martín Catalán y Antón de Villalba; albañiles; el carpintero fue Bautista de Escobar y el escultor Pedro Rosales"15. Sin embargo, Blázquez no menciona ningún detalle más que pudiera aparecer en el documento, dejando en el aire aspectos tan importantes como la cantidad en que se tasaron las obras o la persona que realizó el proyecto ${ }^{16}$.

Los primeros datos que hemos encontrado en torno a la construcción de este edificio aparecen en la carta de obligación que otorgó Francisco Hernández Hurtado, cantero vecino de Villanueva de los Infantes, el 15 de junio de 1628, ante Francisco Flores, escribano público de Almagro. En este documento, Francisco se comprometía a sacar toda la piedra que fuese necesaria para levantar la ermita. En concreto, el texto estipulaba que, en el plazo de dos meses a contar desde oy dia de la fecha, habría de sacar (...) quinientas carretadas de piedra de manposteria gruesa y de cuerpo de la cantera y canteras que ay desde la guerta de don Juan de Coca vecino desta villa que esta en el pago de Mirabuenos hasta la hermita de San Andres (...) sin que tenga obligacion de otra cossa mas de ayudar a cargar la dicha piedra estando en la dicha cantera. Además, el maestro se comprometió a que dentro de ottro mes despues de cumplidos los dos de suso declarados que sera a quinze dias del mes de setiembre deste pressente año ansimismo sacare toda piedra (sic.) que fuere nezesaria para los coclos de la capilla mayor de Nuestra Señora de las Nieves que edifica su eselençia el señor don Alvaro de Bazan Marques de Santa Cruz y estos zoclos an de tener los mayores una bara de largo y el que menos tres quartas de media bara de alto cada uno y dos terçias de lecho los quales tengo de labrar sacar y asentar en la dicha capilla a mi costa dandome peones y un maestro de arbañil que me ayude a ello ${ }^{17}$.

Hasta este momento, el documento no menciona en ninguna ocasión a Esteban Perolli, pero su nombre aparecerá cuando se detallen las cantidades que debía percibir Francisco Hernández Hurtado por su trabajo. Sobre este asunto, el cantero precisaba que por rrazon dello qual se me a de dar y pagar por Estevan de Perola pintor y Juan Cansino en nombre del dicho señor Marques de Santa Cruz por cada una de las dichas quinientas carretadas de piedra que ansi tengo de sacar de manposteria a dos rreales cada una que montan mil rreales y por cada bara de los

${ }^{14}$ Según Díez de Baldeón la idea "comenzó a materializarse el 5 de diciembre de 1629, fecha en la que fue anunciada la subasta de las obras del nuevo santuario". Además, y por lo que respecta al desarrollo de su fábrica, afirmó que "el proyecto permaneció parado pues la construcción no comenzó hasta abril de 1637", precisando que la iglesia se terminó en 1641 (DíEz De BALdeón, 1993: 263). El edificio se caracteriza por su sobriedad y sencillez. Se trata de un recinto con planta de cruz latina, nave única dividida en cuatro tramos y cabecera plana. El crucero se cubre con una cúpula sobre pechinas, mientras que el resto de los espacios (el presbiterio, los brazos del crucero y la nave) se cierran con bóvedas de medio cañón con lunetos. Sus muros, además, se articulan mediante pilastras toscanas que tienen los capiteles integrados en el entablamento que recorre el interior.

15 Blázquez Mateos, 1999: 113.

16 Eduardo Blázquez tampoco aporta muchos detalles sobre este documento, por lo que no sabemos si se trata de la escritura original o de una simple referencia a la misma que aparecía en el legajo que consultó.

${ }_{17}$ Carta de obligación otorgada por Francisco Hernández Hurtado ante Francisco Flores, el 15 de junio de 1628. AHPCR, Protocolos, legajo 1666, sf.

Arch. esp. arte, LXXXVII, 346, ABRIL-JUNIO 2014, 171-178

ISSN: 0004-0428, eISSN: 1988-8511, doi: 10.3989/aearte.2014.13 


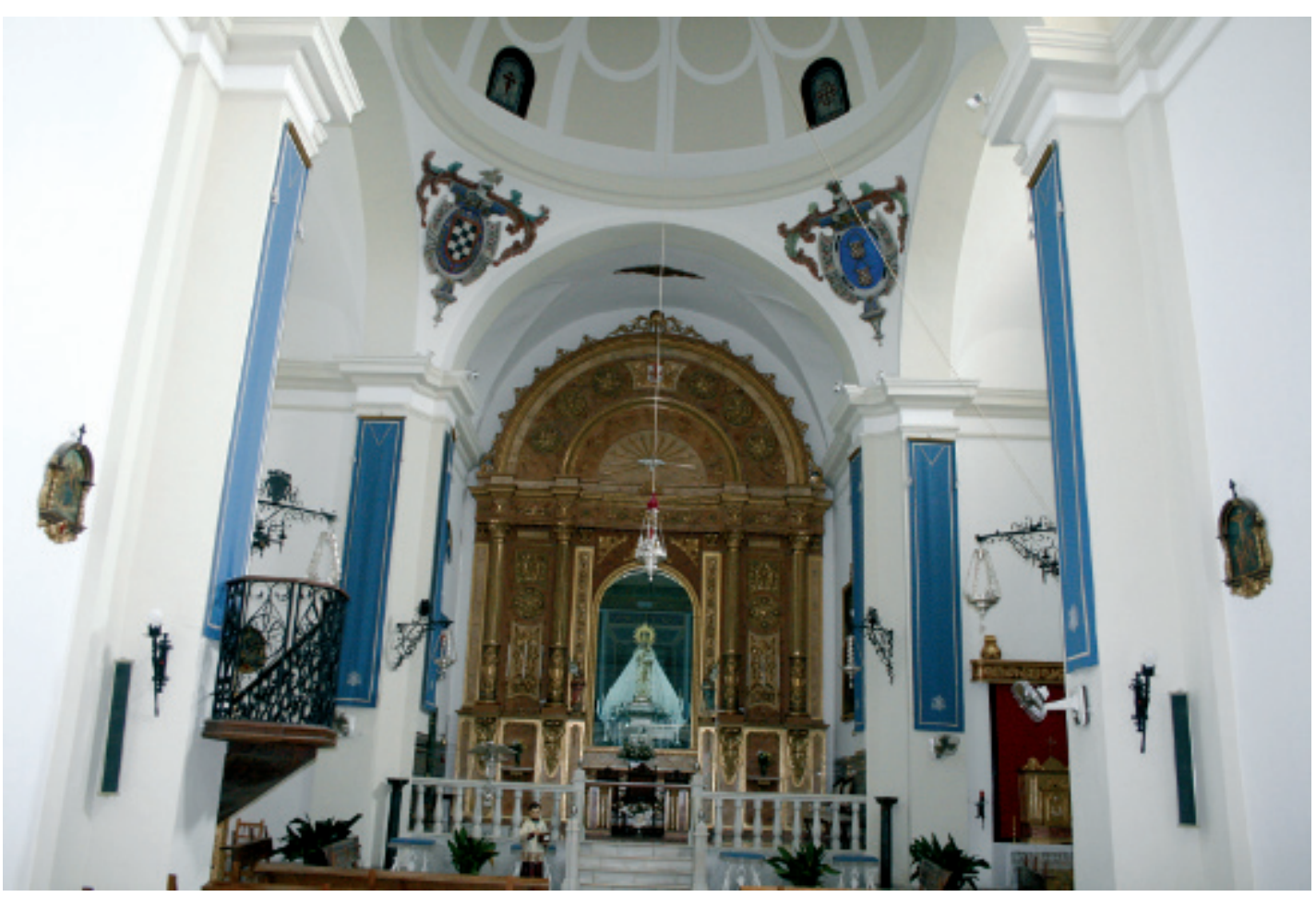

Fig. 1. Interior de la ermita de Nuestra Señora de las Nieves, en Almagro.

dichos zoclos y esquinas sacados labrados y asentados como dicho es a nuebe rreales por cada bara $^{18}$.

La escritura no hace ninguna referencia a la labor como artista de Esteban, salvo la breve mención a su condición de pintor, pero en cambio nos informa sobre el papel que desempeñaba como representante del Marqués de Santa Cruz en este tipo de asuntos. Esteban sería el encargado de pagar al cantero por su tarea y, como tal, estuvo presente en la redacción del documento, dejando estampada su firma en él.

Al igual que nos ocurrió con Juan Esteban, la siguiente referencia que hemos podido localizar sobre el segundo miembro de la familia es la más importante desde el punto de vista artístico, porque nos permite adjudicar la autoría del proyecto de reforma en cuestión a Esteban Perolli. El dato aparece en una escritura de fianza otorgada en esta ocasión por Gregorio Martín Guchillero, Juan Martín Catalán y Antón de Villalva, albañiles, y Bautista de Escobar, carpintero, el 13 de febrero de $1629^{19}$. Los maestros, acompañados por sus fiadores, comparecieron ante Francisco

18 Francisco Hernández reconoció haber recibido de Esteban Perolli y Juan Cansino, como cantidad a cuenta, 200 reales que balen seis mill y ochocientos maravedis, precisando que el resto del dinero se le debía pagar como fuere sacando la dicha piedra labrandola y asentandola como dicho es. Carta de obligación otorgada por Francisco Hernández Hurtado ante Francisco Flores, el 15 de junio de 1628. AHPCR, Protocolos, legajo 1666, sf.

${ }_{19}$ Carta de obligación otorgada por Gregorio Martín Guchillero, Juan Martín Catalán y Antón de Villalva, albañiles, y Bautista de Escobar, carpintero, ante Francisco Flores, el 13 de febrero de 1629. AHPCR, Protocolos, legajo 1667, sf. Gracias a este documento, y a otro que utilizaremos más adelante, sabemos que Gregorio Martín no se apellidaba Chillero, como menciona Blázquez, sino Guchillero, diferencia que podría deberse a una pequeña errata del escri- 
Flores, escribano público de Almagro, y tras declarar que por quanto en nos los dichos Gregorio Martin Guchillero y Juan Martin Catalan quedaron y fincaron de ultimo rremate el hazer las obras y rreparos que se an de hazer en la hermita de Nuestra Señora de las Niebes y obra nueba que en ella se a de hazer por horden de su eselençia don Albaro de Bazan Marques de Santa Cruz, se comprometieron a realizar las dichas obras y rreparos (...) a los plazos y segun y de la manera y como se contiene en la plata (sic.) que en rrazon dello esta fecha, especificando que construirían el cruzero capilla mayor sacristia y todo lo demas que se ha de hazer en la dicha hermita. No obstante, lo más importante es que habrían de hacerlo conforme a lla (sic.) traza y condiciones que en rrazon dello estan fechas que estan firmadas de Diego de Morales contador de su eselençia el Marques de Santa Cruz y de Estevan de Perola vecino desta villla de Alma$g^{20}{ }^{20}$. El diseño de la ermita sería, por tanto, obra de Esteban Perolli, por lo que ya podemos dejar de lado las atribuciones que se habían realizado hasta el momento y que, desde un punto de vista estilístico, vinculaban el edificio con la figura de Nicolás de Vergara el $\mathrm{Mozo}^{21}$, una adjudicación que sería del todo imposible si tenemos en cuenta que este maestro, nacido en 1542 , falleció en 1606. Por otra parte, y en relación con las funciones que desempeñaba el maestro como representante de don Álvaro de Bazán, mencionar que Esteban Perolli también estuvo presente a la hora de otorgarse el documento, quedando muy claro que contaba con poder del Marqués de Santa Cruz para realizar este tipo de gestiones ${ }^{22}$.

La obra se remató en 59.500 reales (2.023.000 maravedís $)^{23}$ y el 7 de marzo de 1630 Bautista de Escobar certificaba haber recibido 6.000 reales a cuenta por el trabajo que habrían de ha$\operatorname{cer}^{24}$. Los datos que acabamos de aportar cambian sustancialmente la visión que teníamos hasta

bano o de la transcripción, pero lo más importante es que el protocolo redactado por Francisco Flores no hace referencia a nadie que se llamara Pedro Rosales, ni a ningún escultor.

${ }^{20}$ Carta de obligación otorgada por Gregorio Martín Guchillero, Juan Martín Catalán y Antón de Villalva, albañiles, y Bautista de Escobar, carpintero, ante Francisco Flores, el 13 de febrero de 1629. AHPCR, Protocolos, legajo 1667, sf.

21 Díez De BAldeón, 1993: 263. En su momento Enrique Herrera también planteó la posibilidad de que la ermita fuera diseñada por Esteban, posibilidad que ahora queda certificada. Herrera MALDONADO, 2003: 270.

${ }^{22}$ El documento precisa que a la firma del mismo se hallaron pressentes los dichos dotor frey Dionisio de Mesa y Estevano de Perola a el qual y en nombre del dicho señor Marques de Santa Cruz y en birtud del poder que su (sic.) eselençia tienen a ellos sostituydos por el dicho Diego de Morales su contador ante Jines de Molina escrivano publico desta villa en diez y ocho dias del mes de agosto de mill y seisçientos y veinte y ocho años azevtaron esta escriptura como en ella se contiene y obligaron los juros y rrentas avidos y por aver del dicho señor Marques de Santa Cruz a que les dara y pagara a los susodichos los dichos çinquenta y nuebe mill y quinientos rreales en que se rremataron las dichas obras. Carta de obligación otorgada por Gregorio Martín Guchillero, Juan Martín Catalán y Antón de Villalva, albañiles, y Bautista de Escobar, carpintero, ante Francisco Flores, el 13 de febrero de 1629. AHPCR, Protocolos, legajo 1667 , sf.

${ }^{23}$ Carta de obligación otorgada por Gregorio Martín Guchillero, Juan Martín Catalán y Antón de Villalva, albañiles, y Bautista de Escobar, carpintero, ante Francisco Flores, el 13 de febrero de 1629. AHPCR, Protocolos, legajo 1667, sf.

${ }^{24}$ Escritura otorgada por Bautista de Escobar ante Francisco Flores, el 7 de marzo de 1630. AHPCR, Protocolos, legajo 1668, sf. Por otra parte, las gestiones para realizar las tareas de cantería continuaron el 14 de julio de 1630. Ese día los canteros Vicente de Aldayda, Domingo de Gamecho e Íñigo de Quirielta comparecieron, junto a Gregorio Martín Guchillero, ante Francisco de Flores. Vicente de Aldayda y sus compañeros se comprometieron a sacar (...) toda la piedra que falta por lavrar para esquinas y çoclos y boquillas de la dicha ovra la qual sacaran de la cantera questa en Curralon junto la guerta de Agustin Lopez (...) la qual dicha piedra estando junto a la dicha obra la an de enviar y acoplar en la dicha obra dandoles el dicho Gregorio Martin la cal que fuere menester para ello todo ello sigun y de la manera como esta la demas piedra questa asentada en la dicha obra. El dicho Gregorio Martín Guchillero, por su parte, se comprometió a pagarles ocho rreales por cada vara de largo de la dicha piedra tiniendo media vara de alto por media vara de lecho. Además, Gregorio tendría que entregarles otros dos rreales por cada una de çinquenta carretadas de piedra de manposteria de las que sacaren de la dicha cantera siendo piecas que se pudieren piconar para

Arch. esp. arte, LXXXVII, 346, ABRIL-JUNIO 2014, 171-178

ISSN: 0004-0428, eISSN: 1988-8511, doi: 10.3989/aearte.2014.13 
ahora de la ermita, certificando de paso la asimilación por parte de Esteban Perolli de los planteamientos típicos de la arquitectura cortesana, sobria y austera, del primer tercio del siglo XVII.

\section{BIBLIOGRAFÍA}

Blázquez Mateos, Eduardo, El arte del Renacimiento en la provincia de Ciudad Real, Ciudad Real, 1999.

Bustamante, Agustín y Marías, Fernando, "La estela de El Viso del Marqués: Esteban Peroli", en Archivo Español de Arte, ‥ ${ }^{\circ} 218,1982$, pp. 173-185.

Del Campo Muñoz, Juan, "La familia Peroli y otros italianos en Viso del Marqués (1575-1613)", en Boletín del Museo e Instituto Camón Aznar, N. . 71, 1998, pp. 53-64.

Díez de Baldeón, Clementina, Almagro: arquitectura y sociedad, Toledo, 1993.

Herrera Maldonado, Enrique, "La renovación de los lenguajes artísticos y la contribución de algunos artistas italianos en La Mancha entre los siglos XVI y XVII: los Perolli", en Correspondencia e integración de las artes: 14 Congreso Nacional de Historia del Arte, Vol. 1, Málaga, 2003, pp. 259-271.

Herrera Maldonado, Enrique, "El arte en La Mancha en tiempos de don Quijote", en La monarquía hispánica en tiempos del Quijote, Madrid, 2005, pp. 373-398.

López Torrijos, Rosa, "Juan Bautista Perolli. Obras genovesas. I", en Archivo Español de Arte, N. . 289, 2000 , pp. 1-22.

López Torrijos, Rosa, “Juan Bautista Perolli. Obras genovesas. II", en Archivo Español de Arte, N.․ 298, 2002, pp. 145-165.

López Torrijos, Rosa, “Arte e historia común en el Palacio del Viso", en España y Génova. Obras, artistas y coleccionistas, Madrid, 2004, pp. 129-138.

López Torrijos, Rosa, "De la Superba al Señorío: la vida de las maestranzas genovesas en El Viso durante el siglo XVI", en El arte foráneo en España: presencia e influencia, Madrid, 2005, pp. 51-59.

López Torrijos, Rosa, "Sobre pintores italianos en España [Castello, Perolli y el falso Cesare Arbasia en el Palacio del Viso]", en In sapientia libertas: escritos en homenaje al profesor Alfonso E. Pérez Sánchez, Madrid, 2007, pp. 198-202

López Torrijos, Rosa, Entre España y Génova. El Palacio de Don Álvaro de Bazán en el Viso, Madrid, 2009.

Fecha de recepción: 27-VI-2013

Fecha de aceptación: 24-IX-2013

açeras a la dicha obra tiniendo cada una pieca una terçia de alto. Escritura de obligación otorgada por Vicente de Aldayda, Domingo de Gamecho e Ínigo de Quirielta ante Francisco Flores, el 14 de julio de 1630, Protocolos, legajo 1668 , sf. A la vista de estos datos resulta obvio que la fecha manejada por otros investigadores para datar el inicio de las obras (1637) no era correcta. 